\title{
Minitrack Introduction: Software Development for Self-Adaptation in Services, Wearables, and IoT Devices
}

\author{
Rose Gamble \\ University of Tulsa \\ gamble@utulsa.edu
}

\author{
Matt Hale \\ University of Nebraska - Omaha \\ mlhale@unomaha.edu
}

\begin{abstract}
This minitrack focuses on services, wearables, and IoT devices as targets for self-adaptation, along with the software development methods, techniques and frameworks needed to employ self-adaptation in these domains.
\end{abstract}

\section{Introduction}

Self-adaptive systems research has been steadily emerging as a force behind the development of autonomous systems [1-3]. These systems may support one or more adaptive operations, such as self-healing, self-repairing, self-maintaining, self-coordinating, selfreferencing, and self-securing, and can be applied to a wide variety of systems. Wearable and IoT devices have reached a high level of ubiquity, relying on seamless interactions with web and cloud services, often through the use of intermediate base stations. These devices and services are acquiring and reporting extensive amounts of information, making them prime candidates for self-adaptation. As the devices become more computationally powerful, they will be able to make local decisions regarding this information, as well as rely on the adaptive services with which they communicate to inform those decisions. Situational awareness is a major factor in adapting behavior and functionality as reflected in improvements in decision making, performance, sustainability, security, and usability, but additional techniques are needed to make it accessible by wearable and IoT devices.

The first offering of the minitrack had one paper presented. In "A Conceptual Architecture for Enabling Future Self-Adaptive Service Systems” Burzlaff and Bartelt explore how integration knowledge can be represented and used to support IoT devices and data sources being integrated on-demand to create a flexible architecture.

\section{References}

[1] Software Engineering for Self-Adaptive Systems I, Editors: Cheng, B.H.C., de Lemos, R., Inverardi, P., Magee, J., Springer, 2009.

[2] Software Engineering for Self-Adaptive Systems II, Editors: de Lemos, R., Giese, H., Müller, H., Shaw, M. Springer, 2013.

[3] Software Engineering for Self-Adaptive Systems III, Editors: de Lemos, R., Garlan, D., Ghezzi, C., Giese, H., Springer, 2017. 